\title{
Erratum: Replication stress in MLL-rearrangements
}

\section{Michael Milyavsky, Boris Gole, and Lisa Wiesmüller}

Oncoscience. 2015 Dec 30;2(12):938-9

PMCID: PMC4675780 PMID: 26909360

DOI: 10.18632 /oncoscience.281

In this Editorial, corresponding authorship was listed incorrectly. Authors Michael Milyavsky and Lisa Wiesmüller shared corresponding authorship of this paper. It is corrected as follows:

\section{Corrected correspondence information:}

Michael Milyavsky: Department of Pathology, Sackler Faculty of Medicine, Tel-Aviv University, Tel-Aviv, Israel Correspondence: Michael Milyavsky, e-mail: mmilyavsky@post.tau.ac.il

Lisa Wiesmüller: Division of Gynecological Oncology,Department of Obstetrics and Gynecology, Ulm University, Ulm, Germany Correspondence: Lisa Wiesmüller, e-mail: lisa.wiesmueller@uni-ulm.de 